Char act er i st i cs of el ect roconvect i on turbul ence and proposal of its appl i cat i on to turbul ent transport experi ment in a rot at ing spheri cal shel I

\begin{tabular}{|l|l|}
\hline $\begin{array}{l}\text { journal or } \\
\text { publ i cat i on titl e }\end{array}$ & H gh Energy Densi ty Physi cs \\
\hline vol une & 31 \\
\hline page r ange & $79-82$ \\
\hline year & 2019- 04 \\
\hline URL & ht t p: //hdl . handl e. net /10655/00012839 \\
\hline
\end{tabular}




\title{
Characteristics of electroconvection turbulence and proposal of its application to turbulent transport experiment in a rotating spherical shell
}

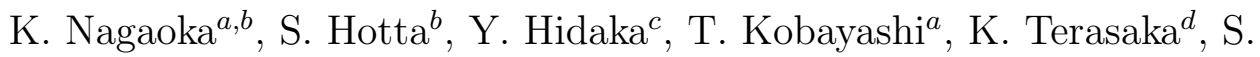 \\ Yoshimura $^{a}$ \\ ${ }^{a}$ National Institute for Fusion Science, 322-6 Oroshi, Toki, 509-5292 Japan \\ ${ }^{b}$ Graduate School of Science, Nagoya University, Furocho, Chikusa, Nagoya, 464-8602 \\ Japan \\ ${ }^{c}$ Department of Applied Quantum Physics and Nuclear Engineering, Faculty of \\ Engineering, Kyushu University, 744 Motooka, Fukuoka 819-0395, Japan \\ ${ }^{d}$ Interdisciplinary Graduate School of Engineering Science, Kyushu University, 6-1 \\ Kasuga-koen, Kasuga 816-8580, Japan
}

\begin{abstract}
An electroconvection (EC) in liquid crystal is used in the experimental study of turbulent transport. The transport characteristics in EC turbulence were investigated with a planer cell, and the diffusive property of turbulent transport was identified, which is identical to that of normal viscus fluid described by Navier-Stokes equation. Experimental research on turbulent transport using EC in a rotating spherical shell is proposed. A demonstration of the production of EC turbulence in a rotating spherical shell was carried out, where the radial gravity was replaced by electric field.
\end{abstract}

Keywords: Turbulent transport, Liquid crystal, electroconvection, Rotating spherical shell

\section{Introduction}

A turbulence in rotating spherical shell attracts much attention from scientists in astrophysics and geophysics[1] because a variety of structure formations are observed on planets. A large scale magnetic field generation in a star such as the Sun is considered to be related to large scale flow generated in the convection zone. Numerical studies to understand large scale flow 
and magnetic field generation have been carried out, and have significantly progressed with development of super computers. However, there remain discrepancies of the parameters between numerical model and astrophysical and/or geophysical objects[2, 3, 4].

On the other hand, experimental studies of turbulence in rotating spherical shell are limited because of the difficulty of realizing of radial gravity in laboratories. One of the symbolic experiments was carried out by F. H. Busse and C. R. Carrigan, and demonstrated the formation of Taylor columns in a rotating spherical shell, in which the turbulence was driven by the centrifugal force and the temperature gradient[5]. In order to minimize the difficulty of the realization of radial gravity, an outstanding experiment was carried out in a space craft [6]. As far as the authors know, experimental study of turbulence in spherical shell with radial gravity has not yet been performed.

In this paper, a new experiment on turbulence in spherical shell using a liquid crystal is proposed. The radial gravity and temperature gradient to drive the turbulence are replaced by only radial electric field. Following sections will describe the experiment of turbulence in liquid crystal. The experimental investigation of turbulent transport characteristics in liquid crystal, and the comparison with those in normal viscous fluids are discussed.

\section{Electroconvection (EC)}

Thermal convection (Reyleigh-Benards convection) is well known as a typical structure formation in a nonequilibrium open system in nature. In laboratories, the thermal convection can be generated in a plane cell heated from the bottom. Thermal instability drives the convection motion, when the heating power is slightly higher than the critical value. One example of experimental set up is shown in Fig.1(a). When the heating power becomes large, the convection changes to turbulence.

On the other hand, an EC is a convection motion of a liquid crystal driven by electric field, which is shown in Fig.1(b). The convection changes to turbulence when the applied voltage becomes much higher. Therefore, the gravity and temperature gradient in the thermal convection can be replaced by the electric field, when the liquid crystal is used in the experiment. The instability in the liquid crystal, which drives the EC, is an interaction between anisotropy of the liquid crystal and current carried by the impu-

rity ions $[7,8]$. When the applied voltage becomes slightly higher than the 

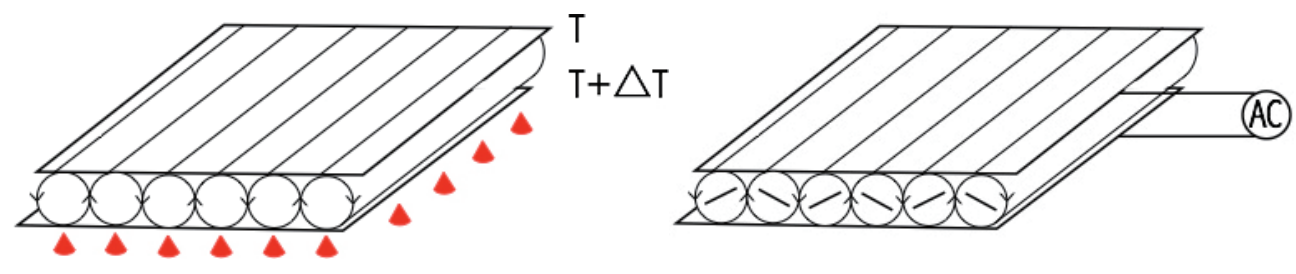

Figure 1: Schematic drawings of thermal convection in a normal fluid and EC in a liquid crystal.

critical value, the thermal fluctuation begins to be enhanced and the convection motion is driven. The reflective index of the liquid crystal also has anisotropy, and the convective motion can be visible due to shadowgraph when one observes the light intensity pattern pass through the liquid crystal. For example, in the case shown in Fig.1(b) the stripe pattern can be seen[9]. The brightness corresponds to the vertical velocity at the position in the liquid crystal. Therefore, one can easily see the two-dimensional flow pattern[10,11], which is an extreme advantage for using liquid crystal in the turbulence experiment.

Another advantage of liquid crystal for turbulence experiment is the controllability of the turbulent state. The voltage control is much easier than the temperature control. In addition, two non-dimensional parameters indicating the state of turbulence, Reynolds number $\left(R_{\mathrm{e}}\right)$ and Prandtl number $\left(P_{\mathrm{r}}\right)$, can be controlled by amplitude $(V)$ and frequency $(f)$ of applied voltage, respectively. It is well known that the linkages are given by

$$
\begin{aligned}
& R_{\mathrm{e}} \propto \varepsilon \equiv \frac{V^{2}}{V_{\mathrm{c}}^{2}}-1 \\
& P_{\mathrm{r}} \propto \frac{f_{\mathrm{c}}}{f},
\end{aligned}
$$

where $\varepsilon$ is a parameter characterizing the turbulent flow, the $V_{\mathrm{c}}$ is the critical voltage at which the convection starts, and $f_{c}$ is the critical frequency of the bias voltage $[12,13]$. The controllability of EC turbulence is also a significant advantage in comparison with normal viscous fluid turbulence experiments.

The liquid crystal has been used in the experiments for investigation of pattern formation and transition to chaos in weak turbulence state[14]. Phase 
diagram of pattern in liquid crystal was compared with that observed in normal viscous fluids, and similarity was discussed in detail[15]. Statistical properties of weak turbulence have been also experimentally investigated using EC[16, 17]. For highly turbulent state of EC, two phases, the so called DSM1 and DSM2 phases, are observed, and transition between them are experimentally investigated[18, 19]. However, the EC itself and the transport properties were not investigated in detail, to the authors' best knowledge. In this sense, the demonstration of normal fluid turbulence using EC is a new experimental challenge to investigate the turbulence properties and turbulent transport.

In our experiment, EC turbulence is produced in plane cells with alternativecurrent bias voltage of $500 \mathrm{~Hz}$. The schematic of the cell is shown in Fig.2 and the thickness of the cell is typically $50 \mu \mathrm{m}$. The nematic liquid crystal (MBBA) fills the cell with surface treatment of homeotropic alignment, in which the liquid crystal molecules aligned perpendicular to the glass plate. The typical critical voltage in this experiment is $V_{\mathrm{c}}=7 \sim 8 \mathrm{~V}$ and the temperature of the cell is kept as $30 \pm 1$ degrees.

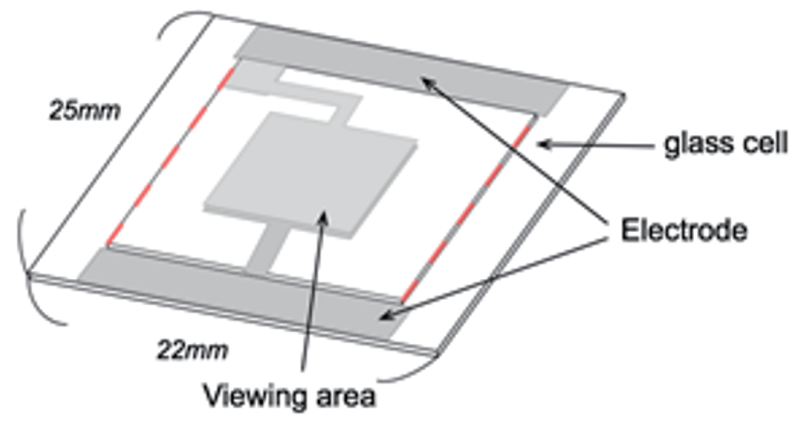

Figure 2: Schematic drawings of plane cell.

\section{Experimental results}

The EC and turbulence are produced in a plane cell. Figure 3 shows two-dimensional pattern of light intensity passed trough the cell, which corresponds to two-dimensional pattern of flow velocity in $z$-direction. In the case of $V / V_{\mathrm{c}}=2$, one can see a stripe, indicating formation of convection roll 


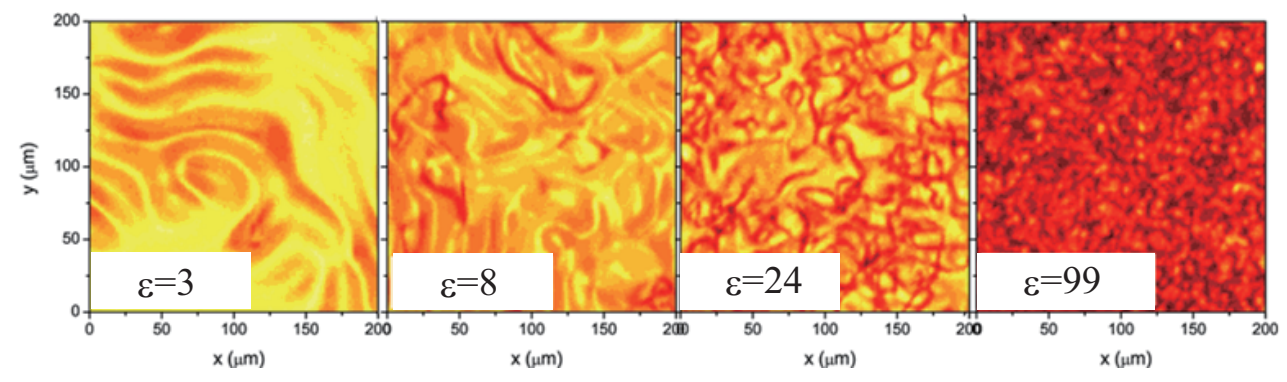

Figure 3: Two dimensional vertical velocity profiles obtained by the shadowgraph technique, and the dependence on $\epsilon$.

in the cell. By increasing bias voltage, the typical scale of the roll becomes smaller and the flow becomes turbulent.

In order to investigate the transport characteristics in EC and turbulence, a particle tracing technique was developed. Small plastic particles with the diameter of $6.5 \mu \mathrm{m}$ and the mass density almost identical to the liquid crystal are distributed in the cell. The particle motion in the $\mathrm{x}-\mathrm{y}$ plane is observed with a CCD camera and visualizes the local turbulent flow with the scale of the particle. It is well-known that the particle tracing technique is applicable to investigate the turbulent transport characteristics[20]. Figure 4(a) shows an example of particle orbit traveled in the EC turbulence. One can see the random particle motion driven by the local turbulent flow. In order to clarify the transport property, the scaling with arbitrary exponent $H$ :

$$
<l^{2}>\propto t^{2 H}
$$

where $l$ is the traveling distance of the particle with the duration time $(t)$. The $<>$ denotes the ensemble average, and $H$ is the Hurst exponent. The cases with $H=1 / 2$ and $H=1$ correspond to classical diffusion and to ballistic transport, respectively. The cases with $0<H<1 / 2$ and with $1 / 2<$ $H<1$ correspond to subdiffusive and superdiffusive transport, respectively. The $H$ values evaluated with the experiment are almost $1 / 2$ as shown in Fig.4(b). Therefore, the transport is diffusive.

The effective diffusivities of particles can be evaluated by

$$
D_{\text {eff }}=\frac{<l^{2}>}{t}
$$

and is shown in Fig.4(c). One can see the increase of effective diffusivity with $\varepsilon$ and linear relation between them in highly turbulent state (corresponding to 

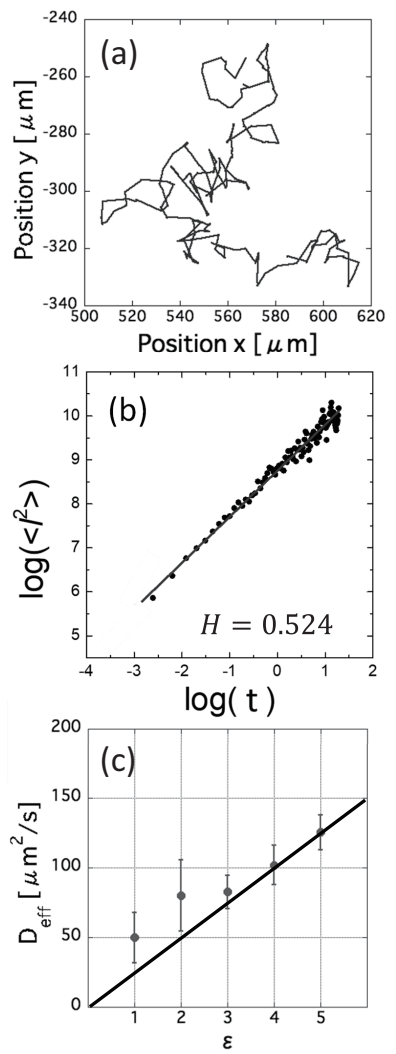

Figure 4: (a) Particle trajectory in EC turbulence, (b) averaged squired-traveling distance as a function of the traveling time, and (c) effective diffusivity evaluated by eq. 4 as a function of $\epsilon$.

large $\varepsilon$ ). In the case of turbulence driven in normal fluids described by NavierStokes equation, the turbulent diffusivity is proportional to the Reynolds number $\left(R_{\mathrm{e}}\right)$, if the molecule diffusivity is fixed. In the highly driven EC turbulence, turbulence and turbulent transport can be linked with those of normal viscous fluids turbulence, if the Reynolds number is replaced with $\varepsilon$ as shown in eq.2.

\section{Discussions and summary}

The EC turbulence is produced in a planar cell, and it is successfully demonstrated that the turbulent diffusivity in the regime of $\varepsilon$ much larger 
than unity is similar with transport characteristics in normal fluid turbulence. Although further comparisons of turbulent transport characteristics between EC turbulence and normal fluid turbulence should be carried out. It is noted that the EC turbulence is a powerful tool to investigate turbulent transport with sophisticated geometries or conditions in which the experimental study with normal fluids is impossible or very difficult.
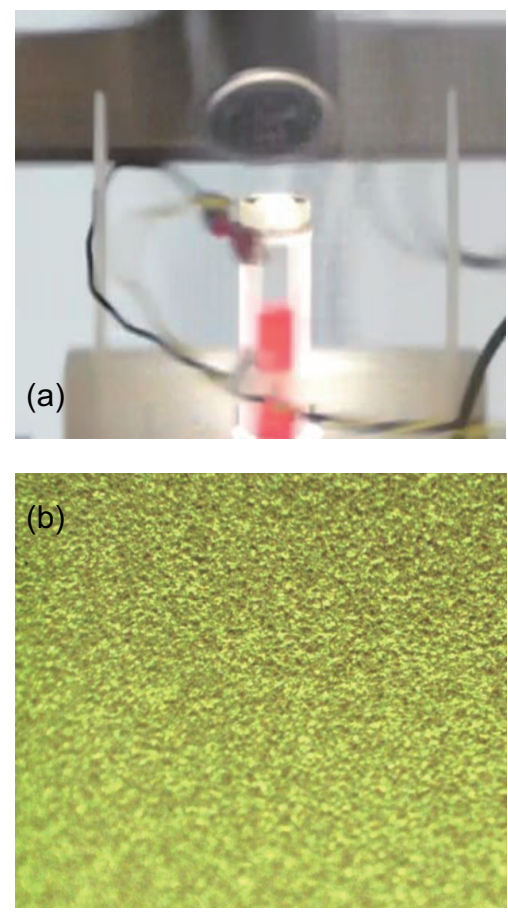

Figure 5: Schematic images of planar cell.

One of the challenging targets is the thermal convection turbulence in rotating spherical shell. The demonstration of production of EC turbulence in one-half of a rotating spherical shell was carried out. Figure 5(a) shows the experimental setup, and Fig.5(b) shows the pattern of transparent light, which corresponds to two-dimensional turbulent flow pattern. The shell was made with pyrex glass, and the spherical surface was manufactured with the accuracy of less than $1 \%$ of the curvature $\left(R_{\text {curv }}=5 \mathrm{~mm}\right)$. The thickness is $50 \mu \mathrm{m}$. The electric field was applied in the radial direction to drive EC turbulence, thus the radial gravity is replaced by the electric field, which is difficult to realize using normal fluids. The turbulence in the spherical 
shell was easily produced and the rotation was also applied up to $4 \pi \mathrm{rad} / \mathrm{s}$. No clear effects of the rotation upon the turbulence were observed in the experimental condition of Rossby number of $R_{\mathrm{o}}>1$. In order to observe the effect of rotation and/or structure formations, further developments such as application of fast rotation and expansion of the view area in spherical surface, etc, are necessary.

In conclusion, turbulence and turbulent transport experiment using an EC turbulence was demonstrated. The simplicity in diagnostics and controllability of EC turbulence are significant advantages for turbulence experiments, and enable replacing radial gravity with electric field for driving turbulence in spherical geometry, such as stars and planets. It is noted that this idea expands the possibility of experimental study to investigate many astrophysical observations, such as differential rotation in the Sun, super rotation in Venus, zonal flow structure and hexagonal pattern formation near the poles in Jupiter, etc.

\section{Acknowledgments}

One of the authors (KN) would like to thank Dr. S Kai (Kyushu Univ.) for support of experimental development and Dr. S. Tsuneta, members

of HINODE (NAOJ) and Dr. M. Nakata (NIFS) for fruitful comments and discussions. This research was supported by NINS Program for CrossDisciplinary Study, NIFS18KBAR020, KAKENHI 23654072, and 26610194.

\section{References}

[1] F. Stefani, A. Gailitis, G. Gerbeth, ZAMM Z. Angew. Math. Mech. 88 930 (2008).

[2] Y. Masada, K. Yamada and A. Kageyama, 77811 (2013).

[3] H. Hotta Science 3541386 (2016).

[4] A. Kageyama et al., Nature 4541106 (2008).

[5] F. H. Busse and C. R. Carrigan, CHAOS, 4123 (1994).

[6] J. Toomre, J. E. Hart and G. A. Glatzmaier, 'The Internal Solar Angular velocity', Chapter 5, pp. 27-44, Springer, 1987. 
[7] E. F. Carr, J. Mol. Cryst. 7253 (1969).

[8] W. Helfrich, J. Chem. Phys. 514092 (1969).

[9] R. William, J. Chem. Phys. 39384 (1963).

[10] K. Kondo,et al, Jpn. J. Applied Phys. 22394 (1983).

[11] S. Rasent, et al., Experiments in Fluid 7412 (1963).

[12] S. Kai K. Hayashi and Y. Hidaka, J. Phys. Soc.Jpn. 40301 (1976).

[13] S. Kai, M. Tanaka and K. Hirakawa, J. Phys. Soc.Jpn. 452051 (1978).

[14] A. Buka and L. Kramer, "Patter formation in Liquid Crystal", Springer (1996).

[15] R. Krishnamurti, J. Fluid Mech. 60285 (1973).

[16] S. Kai, K. Hirakawa and Y. Hidaka, J. Phys. Chem. 10019007 (1996).

[17] Y. Hidaka, et al Phys. Rev. E., 56 R6256 (1997).

[18] S. Kai, W. Zimmermann, M. Andoh and N. Chizumi, Phys. Rev. Lett. 641111 (1990).

[19] K.A. Takeuchi, and M. Sano, et al Phys. Rev. Lett. 104, 230601 (2010).

[20] 'Turbulence and Diffusion', Oleg G. Bakunin, Springer (2008). 